\title{
Development and application of the Imperial College Obesity Strategy Assessment Framework for analysing local obesity strategies
}

\author{
Nik A.A. Tuah ${ }^{1}$, Samrina Qureshi ${ }^{1}$, Waljit S. Dhillo ${ }^{2}$ and Azeem Majeed ${ }^{1}$ \\ ${ }^{1}$ Primary Care and Public Health, School of Public Health, Imperial College London, London, UK \\ ${ }^{2}$ Division of Investigative Science, Faculty of Medicine, Imperial College London, Hammersmith Hospital, London, UK
}

\begin{abstract}
Background: Obesity is a major public health issue because of its increasing prevalence and impact on health. The management of overweight and obesity has been a government priority for many years. However, overweight and obesity management at a local level has often been ineffective. Although there is a need to examine obesity strategies and policies for local populations, there is currently no readily available framework for evaluating local obesity strategies. We therefore developed a framework, the Imperial College Obesity Strategy Assessment Framework (IC-OSAF), for examining the content of local obesity strategies. Methods: We adapted two previous policy analysis frameworks (Bardach's Eightfold Path Framework and Collins' Health Policy Analysis Framework) and used these with information from national guidelines to develop an obesity strategy analysis framework. We then piloted this framework using the obesity strategy for one London primary care trust (PCT). Results: The framework was applied successfully and helped identify limitations and omissions in the PCT obesity management strategy. Conclusions: The IC-OSAF is a practical, easy-to-use tool for the analysis of local obesity management strategies. The framework can help identify gaps and limitations in strategies to help reduce variations in obesity management between PCTs.
\end{abstract}

Key words: healthy policy; obesity; policy analysis; primary care

Received 3 February 2010; accepted 17 June 2010; first published online 12 August 2010

\section{Introduction}

The World Health Organization (WHO) estimated in 2006 that approximately 1.6 billion adults were overweight, and at least 400 million adults were obese and that about 20 million children under the age of five years were overweight (World Health Organization, 2006). In the United Kingdom, the proportion of adults with

Correspondence to: Nik A.A. Tuah, Doctoral Researcher, Primary Care and Public Health, School of Public Health, Imperial College London, Charing Cross Campus, St. Dunstans Road, London W6 8RP, UK. Email: a.tuah08@imperial.ac.uk

(C) Cambridge University Press 2010 obesity has significantly increased in recent decades, with no signs of any reversal in this trend (National Health Services, 2008). The ineffectiveness of interventions for obesity has resulted in predictions that around 12 million adults and 1 million children will be obese in the United Kingdom in 2010. This represents a shift in recent years of around 3.5 million adults who were previously within a healthy weight range or with a body mass index of $<30 \mathrm{~kg} / \mathrm{m}^{2}$ into either the overweight or obese range, respectively, by 2010 (Department of Health, 2006; Pryke and Docherty, 2008). The Foresight Report predicts further increases in obesity and states that by 
$2050,60 \%$ of men, $50 \%$ of women and $25 \%$ of children in the United Kingdom could be obese (Government Office for Science, 2007).

The contributing factors for an increase in overweight and obesity are a global shift in diets towards an increased intake of energy-dense foods (high in fat and sugars). Other factors include decreased physical activity and a sedentary lifestyle; and environmental factors such as changing modes of transportation, a shift from manual to non-manual occupations and increasing urbanisation (World Health Organization, 2006). A number of studies have examined strategies to prevent obesity. Some studies have shown that children are perhaps the most important group to target; however, a majority of these studies were short term. Studies that focused on combining dietary and physical activity approaches generally did not significantly impact on obesity, although they helped in promoting a healthy diet and increased physical activity levels (Summerbell et al., 2005).

The consequences of obesity are significant impairments of health and longevity. Diabetes, heart disease, hypertension, cancer and osteoarthritis are all more common in overweight and obese people (London Health Observatory, 2008). The Foresight Report comments that the risk of hypertension is increased up to fivefold in people with obesity; that coronary heart disease (CHD) risk increased twofold in adults under 50 years of age; and that $10 \%$ of all cancer deaths in non-smokers are associated with obesity. Moreover, around $8.7 \%$ of total deaths in the United Kingdom are estimated to be as a result of excess weight (Banegas et al., 2003). Obesity also reduces quality-adjusted life expectancy by about three years in males and six years in females (Brønnum-Hansen et al., 2007; Pryke and Docherty, 2008). Furthermore, obesity has huge economic implications for the country from direct treatment costs and from indirect costs, such as sickness absence, with estimated total annual costs of $£ 3.5$ billion in 2002 (House of Commons Health Committee, 2004). The Foresight Report also predicts that National Health Service (NHS) costs from overweight and obesity will reach $£ 10$ billion per year by 2050, with the wider societal costs estimated to reach around $£ 50$ billion per year.

Many studies have evaluated the effectiveness of drug, dietary and physical exercise interventions for obesity management among overweight and obese individuals, and have reported moderate weight loss at up to 12 months follow-up (Miller et al., 1997; Padwal et al., 2003; Avenell et al., 2004a; 2004b; Douketis et al., 2005; Jain, 2005; Norris et al., 2005; Nield et al., 2009; Orozco et al., 2008; Shaw et al., 2006). However, there is a lack of evidence about which interventions are effective in the prevention of obesity and overweight or for sustainable long-term weight loss for more than one year in primary care or community settings. Surgical interventions show clinically effective results but are expensive and can be associated with significant complications. Hence, bariatric surgery is reserved for more severe cases (Salem et al., 2005; Picot et al., 2009; Reedy, 2009).

Similar issues were reported in a review of evidence for the management of obesity and overweight: that there is mixed and inconclusive evidence of effectiveness for community-based interventions among adults. Among reported gaps in the evidence are lack of research focusing on the prevention of obesity and overweight; the maintenance of weight loss in adults and children; and for 'upstream' interventions such as policies or strategies at the national or regional level, which focus on population and environmental strategies for the prevention of obesity and overweight (Mulvihill and Quigley, 2003) The failure of obesity management in England may be related to ineffective implementation of obesity strategies and policies at a local population level (House of Commons Health Committee, 2004).

\section{Literature review of national guidelines}

The Department of Health report 'Tackling Obesity in England 2001' highlighted that surgery and drug treatment are potentially effective interventions in the management of overweight and obese patients but are costly and possibly not scalable to a population level. Other interventions include dietary management, physical activity and cardiovascular risk assessment. The National Service Framework (NSF) for CHD and the National Institute for Health and Clinical Excellence (NICE) are other sources of guidelines commonly used by general practitioners in the management of patients with obesity (Comptroller and Auditor General, 2001).

A House of Commons Health Committee report discussed issues in obesity management at 
the primary care level among overweight and obese adults in the United Kingdom (House of Commons Health Committee, 2004). Most contacts with overweight and obese people within the NHS occur in general practice. Hence, general practice offers one setting in which to tackle obesity. However, many obese and overweight patients will present for problems other than their weight and may be reluctant to have this raised by their general practitioner in an unrelated consultation.

Key findings in the House of Commons report include ineffective implementation of obesity guidelines, as well as limited patient access to various obesity treatments such as drugs, behavioural interventions and specialist medical care. The report stated that the main reason for this is that obesity remains a low priority for many service commissioners and providers in the NHS. Furthermore, resources to provide structured, long-term interventions to tackle obesity in primary care were generally unavailable. The report highlighted that ineffective implementation of obesity strategies or policies at the community level is a critical issue for England (House of Commons Health Committee, 2004).

The prevention and management of obesity and overweight has been highlighted in many published reports, including in a 'Cross-Government Health of Nation Strategy' published by the Department of Health in 1992 and in 'Saving Lives: Our Healthier Nation' published in 1999 (Mulvihill and Quigley, 2003). The national obesity policy was heavily scrutinised and criticised for being ineffective in tackling the issue of obesity as reported in the 'Tackling Obesity in England' report in 2001 (National Audit Office, 2001). Evidently, there is a critical gap in the 'upstream' interventions such as policies or strategies at the national or regional level for the prevention of obesity and overweight in both children and adults (Mulvihill and Quigley, 2003).

Currently, there is no readily available framework for evaluating obesity strategies at a local population level in England even though such a framework may be very beneficial in highlighting deficiencies in local strategies. Imperial College Obesity Strategy Assessment Framework (ICOSAF) is developed mainly because the existing policy analysis frameworks are very generic and not tailored or detailed enough to evaluate local obesity strategies. The focus is on obesity strategies for prevention and management among children and adults as emphasised in various UK government reports in the past few years. In this paper, we describe the development and application of such a framework. The objectives of this work were to:

a) review current policy analysis models;

b) develop a new strategy framework (which we have called the IC-OSAF that is suitable for evaluating obesity strategies at a local population level);

c) apply the IC-OSAF to assess the obesity strategy of Primary Care Trusts in London;

d) identify potential policy issues related to obesity and its related health outcomes in this population and provide recommendations to local commissioners.

\section{Methods}

WHO defines health policy as an agreement or consensus on the health issues, goals and objectives to be addressed, with a set of priorities among those objectives and main directions for achieving them (World Health Organization, 1999). Policy analysis is a generic name for a range of techniques and tools to study the characteristics of established policies, how the policies came to be and what their consequences are (Rodriguez-Garcia, 2000). Policy analysis is also considered as both a descriptive activity that dissects and describes how policy is formulated; it is also prescriptive, aiming to influence and change policy making (Ham, 1990). Therefore, it is imperative to distinguish between the analysis of policy process and the analysis of policy content in the policy analysis approach. The main distinction is that process analysis focuses on policy formulation, while content analysis focuses on the substance of the policy (Ham, 1990; Collins, 2005). The above terms will be adapted in course of the content policy analysis approach in this project.

\section{Review of policy models}

The policy analysis phase starts with reviewing the existing policy frameworks or models that are relevant to the scope of this project. The policy analysis models and guidelines for obesity management reviewed were the Health Impact Assessment framework, NICE guidelines, UK

Primary Health Care Research \& Development 2011; 12: 83-94 
Obesity Tool Kit, WHO Global Strategy on Diet, Physical Activity and Health (WHO DPAS), Bardach's 'Eightfold Path Framework' (EPF) and Collins' Health Policy Analysis Framework (HPAF). The most applicable frameworks used in the development of the 'Obesity Strategy Analysis Framework (OSAF)' were Bardach's EPF, Collins' HPAF and NICE guidelines. They were selected as the basis of the framework and are among the widely used frameworks in policy analysis, while NICE is the standard setting body for England's NHS.

\section{Bardach's EPF}

Bardach's 'Eightfold Path' is a generic decisionmaking tool often used to analyse the content of policy strategies, particularly for public policy analysis that can be undertaken by policy analysts in a relatively short time frame. The framework consists of eight steps: (1) define the problem - in an evaluative form and quantified if possible to calibrate the magnitude of the issue; (2) assemble evidence - involves reviewing documents and literature, using statistics as well as interviewing people; (3) construct the alternatives - refers to making a list of all the alternative strategies of intervention to solve or mitigate the problem; (4) select the criteria - commonly used evaluative criteria are efficiency, equality, equity, fairness, justice, freedom, community and process values. Practical criteria that are commonly used include legality, political acceptability as well as robustness and improvability; (5) project the outcomes - anticipating for each of the alternatives on the current list realistic and relevant outcomes that are important to the analyst; (6) confront the trade-offs - a process when one of the policy alternatives under consideration is expected to produce a better outcome than any of the other alternatives; then one must clarify the trade-offs between outcomes associated with different policy options for the sake of the client; (7) decide - refers to checking on how well the analysis is done up to this stage and decide what to do next. At this point, the analyst must be able to convince himself or herself of the plausibility of some course of action; otherwise, one will not be able to convince the client or stakeholders; (8) tell your story - the policy analyst attempts to explain the basic idea

Primary Health Care Research \& Development 2011; 12: 83-94 of the best chosen alternatives in satisfactorily simple and down-to-earth terms. Although the framework offers a step-by-step guidance for policy analysis, these steps do not have to be taken rigidly or in the exact order, and nor are all of them necessarily significant in every problem (Bardach, 2005).

\section{Collins' HPAF}

Collins' HPAF differs from Bardach's 'EPF' as it is designed specifically for health policy analysis mainly used by policy makers who need to make decisions on health issues. According to Collins, health policy analysis is a political as well as social activity and can be very time consuming. Policy makers may face having to make critical decisions in a very short period of time. Therefore, a simplified framework for health policy analysis, which is practical, less time consuming and less resource intensive, is needed in conducting policy analysis studies. Collins' HPAF modifies Bardach's 'EPF' further into the following steps: (1) define the context, (2) state the problem, (3) search for the evidence, (4) consider different policy options, (5) project the outcomes, (6) apply evaluative criteria, (7) weight the outcomes and (8) make the decision. There are two distinctive features for Collin's HPAF, that is, looking at different existing policies before making decisions and making use of evaluation measures in the analysis (Collins, 2005).

\section{Imperial College Obesity Strategy Analysis Framework}

IC-OSAF is a new framework developed from adapting Bardach's EPF, Collins' HPAF and NICE guidelines. Bardach's EPF is the underpinning theory of IC-OSAF, whereas Collins' HPAF, which evolves from Bardach's EPF, provides IC-OSAF with health policy analysis context and components (particularly evaluative and evidence-based approaches). IC-OSAF has adapted concepts from both frameworks and used NICE guidelines as the benchmark in the analysis criteria. Examination of local data (step 3) and current strategies (step 4) are the two main items added in the framework to make it more applicable to obesity and health policy analysis approaches at the community level in the context 


\begin{tabular}{|c|c|c|}
\hline $\begin{array}{l}\text { Bardach's EPF } \\
\text { Define the problem }\end{array}$ & $\begin{array}{l}\text { Collin's HPAF } \\
\text { Define the context }\end{array}$ & $\begin{array}{l}\text { IC-OSAF } \\
\text { State the problem }\end{array}$ \\
\hline Assemble evidence & State the problem & Define the context \\
\hline Construct the alternatives & Search for the evidence & $\begin{array}{l}\text { Search the evidence and } \\
\text { examine the local data }\end{array}$ \\
\hline Select the criteria & $\begin{array}{l}\text { Consider different policy } \\
\text { options }\end{array}$ & $\begin{array}{l}\text { Examine current } \\
\text { strategies }\end{array}$ \\
\hline Project the outcomes & Project the outcomes & Project the outcomes \\
\hline Confront the trade-offs & Apply evaluative criteria & Evaluation \\
\hline $\begin{array}{l}\text { Decide } \\
\text { Tell your story }\end{array}$ & $\begin{array}{l}\text { Weight the outcomes } \\
\text { Make the decision }\end{array}$ & $\begin{array}{l}\text { Make conclusion on value } \\
\text { of local strategies and } \\
\text { provide recommendations }\end{array}$ \\
\hline
\end{tabular}

Figure 1 Evolution of IC-OSAF.

of the United Kingdom. The evaluation process (step 6) is modified according to criteria required by NICE guidelines and health programme evaluation approaches. IC-OSAF distinctively has reformed the final step of the framework by considering the existing local strategies as well as evidence from the literature and other official reports as the basis to decide on the value and generate recommendations for the policy. The comparison of the three given frameworks is shown in Figure 1.

IC-OSAF is a practical content policy analysis framework that can be used by policy analysts to assess obesity-related strategies or policies in a relatively short time frame. The main components of the model (Figure 1) are:

- Seven steps that are reiterative rather than linear: (1) state the problem, (2) define the context, (3) search the evidence and examine the local data, (4) examine current strategies, (5) project the outcomes, (6) evaluation and (7) make conclusions on the value of local strategies and provide recommendations. The definitions and terms of the framework are based on
Bardach's and Collins' frameworks as well as NICE guidelines.

- The analysis criteria are generated from NICE guidelines that made up the characteristics of the framework.

- Use of a wide range of primary and secondary evidence (including published literature, epidemiologic data and clinical evidence).

- Focus on prevention and lifestyle modification strategies at a local population level, as well as on treatment strategies.

\section{Results}

\section{Applying IC-OSAF}

Having developed the IC-OSAF, the next stage was to pilot this framework on the obesity strategy of one primary care trust (PCT). Hammersmith and Fulham (H\&F) PCT in London was selected to pilot the IC-OSAF as this is our local PCT. The description of the framework is shown in Box 1. The framework will also be used to assess obesity strategies of other PCTs in North West London, but the results are not included in

Primary Health Care Research \& Development 2011; 12: 83-94 


\section{Box 1 The description of imperial college obesity strategy analysis framework (IC-OSAF)}

\section{Step}

1) State the problem
2) Define the context

\section{Characteristics}

State the national trends and prevalence of obesity; prevalence of obesity andhealth impact of obesity in PCT.

Describe the profile of the PCT: background information and determinants of health problems (including demography, socioeconomic profile, ethnicity, health, morbidity andmortality indicators)

3) Identify local data andevidence used (then examine where necessary)

4) Examine current strategies

Does the PCT use information from local/national published data and evidence from literature (publications, policy documents, unpublished reports and survey data? yes/no

- NICE guidelines (NICE, 2006) yes/no

- GP data (local and national) yes/no

- UK National Statistics yes/no

- Public Health Report yes/no

- National policy guidelines yes/no

a) What Management strategies are available (based on NICE)? Please circle your answer for each item. NHS:

- develop/implement local obesity strategies yes/no

- specific training yes/no

- develop/implement well-being programmes yes/no

- conduct health impact assessments yes/no

b) What services are available (based on NICE)? Please circle your answer for each item.

NHS:

- primary care yes/no

- community care yes/no

- secondary care yes/no

- tertiary care yes/no

Does PCT have local authorities and partners in the community services?

- early years settings yes/no

- schools yes/no

- workplaces yes/no

- self-help programme yes/no

- commercial programme yes/no

Clinical/Treatment Pathways (Please circle your answer for each item.)

Children:

\begin{tabular}{ll} 
Assessment & yes/no \\
Measurements & yes/no \\
Referral to specialist & yes/no \\
Counselling & yes/no \\
Lifestyle & behavioural/diet/physical activity/family \\
\hline
\end{tabular}


Box 1. Continued

5) Define the outcomes

6) Evaluation

7) Make conclusions about the value of local policies
Drug treatment (not for children younger than 12 years, except under specialist paediatric settings) yes/no

Follow-up yes/no

Adult:

$\begin{array}{ll}\text { Assessment } & \text { yes/no } \\ \text { Measurement } & \text { yes/no } \\ \text { Referral to specialist } & \text { yes/no } \\ \text { Counselling } & \text { yes/no } \\ \text { Lifestyle } & \text { behavioural/diet/physical activity/family } \\ \text { Drug treatment } & \text { yes/no } \\ \text { Surgical treatment } & \text { yes/no } \\ \text { Follow-up } & \text { yes/no }\end{array}$

c) Does PCT have non-NHS public programmes? yes/no

d) Does PCT consider different services and aspects (epidemiological, clinical and economic) for interventions? yes/no (give details if applicable)

e) Do services reflect the ethnic and socio-economic diversity of the PCT? yes/no (give details if applicable)

f) Compare services against the evidence. Do the services offered by PCT have evidence to show they are likely to be effective? yes/no (give details if applicable)

a) Are the key outcome indicators for interventions defined? yes/no short term yes/no intermediate yes/no long term yes/no

b) Are the projected outcomes based on NICE guidelines? yes/no

c) What interventions are implemented by PCT?

d) Any other alternative interventions considered? yes/no (give details if applicable)

e) Is there evidence from the literature supporting the interventions/outcomes stated? yes/no

What plans does the PCT have (if any) to evaluate obesity interventions?

What time frames are specified?

What methods will be used for evaluation?

What are the total costs of the obesity strategy?

Are the costs of the strategy and resources for evaluation clearly identified?

a) Could this PCT's policy be improved? If so, how?

b) Are there PCTs that are examples of good practice?

c) What could be done to improve the evaluation of local policies? 


\section{Box 2 Recommendations for the H\&F PCT obesity strategy}

The policy can be improved by considering the following points:

- Need to clearly define the problem and its significance in H\&F.

- Baseline statistics, such as prevalence of obesity and obesity-related illnesses in H\&F, need to be included in addition to national statistics.

- Additional information is required on the relationship between obesity and demographic variables specific to H\&F. The PCT's strategies should reflect the ethnic and socio-economic diversity of its patients.

- Specific training needs should be identified to efficiently implement new strategies.

- The PCT should consider providing channels for patients to access the adult obesity pathway, for example, devising strategies to address obesity in workplaces, or via self-help, commercial and community programmes. The PCT should also include non-NHS public programmes, if available.

- It should include more details of the exact methods the PCT will use to achieve its strategy.

- The proposed strategies should be evidence-based and the report should demonstrate this with evidence from up-to-date literature.

- Specific, measurable targets and outcomes need to be decided on and clearly stated in the strategy. Including intermediate targets would create a stepwise progression towards achieving the overall aim.

- An evaluation process should be agreed on, as well as when this is performed.

this paper as the preliminary findings are similar to those for H\&F PCT.

The H\&F PCT obesity strategy was examined using IC-OSAF and data analysed by two researchers (NT \& SQ). Any discrepancies were resolved by consensus, or by consultation with a third observer (AM). The purpose of this was to validate and enhance reliability of the framework as well as minimise assessor bias on the findings. IC-OSAF will be continuously fine-tuned as the process of obesity policy analysis is undertaken for other PCTs in the future. The result of this current process is a validated narrative review on the content of the H\&F PCT's obesity strategy, which is summarised in Box 2.

\section{Narrative review of the H\&F PCT's obesity strategy}

Identifying the level of obesity and the broad range of factors that influence its prevalence in $\mathrm{H} \& \mathrm{~F}$ is essential to putting the problem into context. Descriptive data including the local population's vital statistics such as births, deaths by age and sex, and cause of death, as well as health statistics such as morbidity by cause and severity, outcome data and burden of disease data are crucial in defining the problem.

It is important to differentiate between prevalence of obesity in adults and children, as the interventions for them will differ. The H\&F PCT's obesity strategy contains adequate statistics of childhood obesity, derived from the National Child Measurement Programme. This has the advantage that direct comparisons can be made between the H\&F PCT and London/National results. The strategy may benefit from including data on the size of the obesity population being served in $\mathrm{H} \& \mathrm{~F}$, as this is critical for effective planning. There was a lack of information on local obesity prevalence.

In addition to current figures, using statistical methods to project future prevalence and trends of obesity in the PCT would also aid in highlighting the potential magnitude of the problem. There is no information included in the H\&F PCT obesity strategy to demonstrate the health impact of obesity in H\&F. For example, Type 2 diabetes, stroke and CHD can all arise as a consequence of obesity and could be included and then compared to national averages.

Finally, the strategy identifies that there are differing levels of obesity in the various ethnic 
groups within the H\&F PCT. A comprehensive profile of the PCT would put health policy into context and enable an understanding of the specific sociocultural determinants of obesity in the PCT. This would create a shared understanding of the relationship between key factors influencing the levels of obesity and their relative importance. This information could then be used to identify and tailor effective interventions, as well as allowing efficient distribution of resources. Additional information on the relationship between obesity and other demographic variables such as income, disabilities and employment status is needed.

The current H\&F PCT's obesity strategy identifies that a multi-focused approach is required in tackling obesity, and that a balance must be sought between population level measures and more targeted interventions aimed at individual patients. Thus, the strategy has been divided into preventing obesity (by promoting healthy eating behaviours and encouraging physical activity) and treating obesity (adult and child care pathway). The main weakness that IC-OSAF has identified in H\&F's obesity strategy is that it fails to declare the specific methods that will be utilised to execute these strategies.

The H\&F PCT's obesity strategy is based on guidelines from local and national published data, including NICE guidelines, Department of Health publications such as Healthy Weight, Healthy Lives: a tool kit for developing local strategies 2008, primary care data, UK National Statistics and national policy guidelines such as the Foresight Report 2007. However, the amount of published data and evidence incorporated into the H\&F PCT's obesity strategy is very limited. Furthermore, there is no evidence included to support the effectiveness of combating obesity specifically by dietary and physical activity interventions. In addition, the child obesity care pathway is aimed at children between 7-13 years old only; no future plans to prevent, identify and target obesity at an earlier stage are included. The H\&F PCT's obesity strategy would benefit from including supportive statements from recent medical literature.

A multidisciplinary approach to the problem of obesity is demonstrated, and clear indications and detailed pathways for referral stated. The obesity strategies will be implemented via primary and secondary care. Tertiary care services should also be included, if available. Other than for some work with schools, the H\&F PCT obesity strategy does not demonstrate that the PCT has the local authority and community services as partners. The strategies may be unsuccessful if they fail to have sufficient impact because they do not offer the range and depth of the interventions needed. Although self-help and community initiatives are available in $\mathrm{H} \& \mathrm{~F}$, no mention of these is made in the H\&F PCT's obesity strategy (eg, Fit for Life is a 13-week weight management programme consisting of nutrition education, behaviour change therapy and physical activity. Community nutrition project works with community groups to improve knowledge of food and nutrition along with practical skills like cooking and shopping (NHS H\&F, 2009). There are no non-NHS programmes described in the H\&F PCT's obesity strategy and therefore the availability of these services, which are recommended by NICE guidelines (NICE, 2006), is unknown.

Although the H\&F PCT's obesity strategy acknowledges the ethnic and socio-economic diversity of the PCT, it is unclear if these have been considered in the services intended to be offered. This is compounded by the fact that the specific methods and programmes to tackle obesity have not been included. The options available should reflect the ethnic specificity and experience of the nation and be scientifically justified and based on international experience (Collins, 2005).

The H\&F PCT's obesity strategy identifies that additional training will be required to implement the proposed obesity strategy effectively. Although broad training needs within the child obesity care pathway are identified, no specific needs assessment has been performed. No specific training needs have been identified within the adult obesity care pathway. This will be required across the multidisciplinary team due to the likely increase in subjects entering the pathways as identification of obese individuals improves.

The likely costs (including breakdown) involved in implementing the adult and child obesity care pathways are clearly delineated. The H\&F PCT's obesity strategy also appropriately recognises that the obesity pathways will need to be aligned with other care pathways, for example, for cardiovascular disease to avoid confusion and duplication. This will ultimately also have a cost benefit. 
General policy outcomes have been suggested in the H\&F PCT's obesity strategy such as targets to reduce obesity in year six children to levels seen in 2000 by 2020 , and a reduction of premature mortality for cardiovascular disease and cancer, but the report does not specify the exact targets expected to be achieved. The interventions proposed to achieve these outcomes are aimed at dietary and physical activity modification; there are no alternative interventions considered at this stage. However, it can be questioned whether the interventions are based on evidence from current literature. Many studies found that drugs, dietary and physical exercise intervention may result in modest weight loss $(2-5 \mathrm{~kg})$ after one to two years of interventions (Padwal et al., 2003; Douketis et al., 2005). Nevertheless, a clinical review argued that the reported weight lost can be statistically significant, but it may not be clinically significant enough to improve patient's health or quality of life (Jain, 2005).

Both short- and long-term outcomes are included in the H\&F PCT's obesity strategy. This is important as the need for short-term action and impact must be balanced against the drive for longer-term sustainable change (Kopelman et al., 2007). No intermediate outcomes are included; smaller time-specific deliverables within the strategy should be stated to ensure timely progress. Furthermore, stating an estimated end point adds an appropriate sense of urgency and ensures that the objectives do not extend over an unreasonably long time scale. Once objectives have been achieved, the policy objective can extend to maintaining results. The H\&F PCT's obesity strategy does not allude to the achievability of the aims, for example, does the PCT have personnel with the required skill sets, have enough resources and have management support?

Crucially, the need for evaluation is identified in the document. The practicalities of the strategy will be evaluated in a few general practices before widespread implementation. This is important as it will improve efficacy in terms of final outcomes and costs. However, the document does not include information on using systematic data collection methods and building in a robust evaluation process of the outcomes; this is vital to ensure ongoing refinement of the policy. Furthermore, the report should contain details of when, how and by whom this evaluation will be conducted.

Primary Health Care Research \& Development 2011; 12: 83-94

\section{Discussion}

\section{Summary}

There is a strong case to examine local obesity strategies and policies in England due to the established critical gap in the implementation of policies related to obesity management (Mulvihill and Quigley, 2003). Studies on 'upstream' interventions, particularly evaluation of policies and their impact (policy analysis), may fill in the gaps on the 'failure' of conventional obesity management interventions. Most frameworks proposed in the health policy literature use particular concepts and models to explain health policies in abstract, theoretical terms and focus mainly on the macro-analysis of the political system, including the role of the state (Collins, 2005). The IC-OSAF is a practical tool used to analyse the content of obesity strategies implemented at the community level (PCT), which has advantages over other frameworks. It is a practical and quick assessment tool as well as less resource intensive for policy analysts or policy makers. It focuses on community rather than national-level policies; this is where most issues arise in policy planning and implementation.

\section{Context}

IC-OSAF is an evidence-based framework derived from existing decision-making and policy analysis models and national guidelines (Bardach's EPF, CHPAF and NICE UK). It has evolved, been tested and produced results for obesity strategies implemented for the H\&F PCT. Preventive as well as clinical interventions are covered in the content policy analysis, as both are important in dealing with the rising prevalence of overweight and obesity.

\section{Strengths and limitations}

It allows the identification of any potential and actual policy content issues, for example, lack of baseline statistics and details that reflect on the relationship between obesity and demographic variables. The framework enables users to examine and ensure that strategies are completed according to national guidelines, which are crucial for the effective implementation of obesity strategies at the local level. 
The main limitation of the IC-OSAF is that its usage might be limited to UK policy priorities in tackling obesity at the primary care level. Therefore, it is necessary to take into account the relevant existing national guidelines in applying the ICOSAF in countries other than the United Kingdom. The prior assumption is that users have a basic knowledge of the terms used in the IC-OSAF.

\section{Implications}

Hence, the IC-OSAF might be a useful tool for the policy analysts to examine the content of obesity policy within a short time frame and is inexpensive. As a consequence, the findings will enable policy makers to make more rapid decisions and set up relevant strategies or priorities. For England, the ineffective implementation of obesity strategies or policies at the community level is a critical issue (House of Commons Health Committee, 2004), and has been a national governmental concern for many years (Mulvihill and Quigley, 2003). The framework will be further refined from application to obesity strategies from other PCTs and from feedback from users, which generates more research in this area.

\section{Conclusions}

IC-OSAF is a practical and easy-to-use tool for analysing local obesity management strategies. Although national obesity policies exist, obesity continues to be a growing health concern. Most PCTs have publicly stated policies on combating obesity locally, but there is enormous variation between areas. Applying IC-OSAF to these strategies will highlight any shortcomings in them and lead to greater standardisation of the content of policies between PCTs.

\section{Acknowledgements}

We thank the H\&F PCT for supplying their obesity strategy for assessment and Dr Balvinder Kaur for comments on the framework.

\section{Funding}

Nik Tuah is funded by the Government of Brunei. Samrina Qureshi was funded by the London
Deanery. Waljit Dhillo is funded by an HEFCE Clinical Senior Lecturer Award. This project was supported by the NIHR NW London CLAHRC Programme and the Public Health Action Support Team. Imperial College London is grateful for support from the NIHR Biomedical Research Centre Scheme.

\section{References}

Avenell, A., Brown, T.J., McGee, M.A., Campbell, M.K., Grant, A.M., Broom, J., Jung, R.T. and Smith, W.C.S. 2004a: What are the long-term benefits of weight reducing diets in adults? A systematic review of randomized controlled trials. Journal of Human Nutrition \& Dietetics 17, 317-35.

Avenell, A., Brown, T.J., McGee, M.A., Campbell, M.K., Grant, A.M., Broom, J., Jung, R. and Smith, W.C.S. 2004b: What interventions should we add to weight reducing diets in adults with obesity? A systematic review of randomized controlled trials of adding drug therapy, exercise, behaviour therapy or combinations of these interventions. Journal of Human Nutrition \& Dietetics 17, 293-316.

Banegas, J.R., Lopez-Garcia, E., Gutierrez-Fisac, J.L., Guallar-Castillon, N. and Rodriguez-Artalejo, F. 2003: A simple estimate of mortality attributable to excess weight in the European Union. European Journal of Clinical Nutrition 57, 201-208.

Bardach, E. 2005: A practical guide for policy analysis: the eightfold path to more effective problem solving, second edition. Washington, United States of America: CQ Press.

Brønnum-Hansen, H., Juel, K., Davidsen, M. and Sørensen, J. 2007: Impact of selected risk factors on quality-adjusted life expectancy in Denmark. Scandinavian Journal of Public Health 35, 510-15.

Collins, T. 2005: Health policy analysis: a simple tool for policy makers. Public Health 119, 192-96.

Department of Health. 2006: Forecasting obesity to 2010. Retrieved 24 July 2008 from http://www.dh.gov.uk/en/ Publicationsandstatistics/DH_4138630

Douketis, J.D., Macie, C., Thabane, L. and Williamson, D.F. 2005: Systematic review of long-term weight loss studies in obese adults: clinical significance and applicability to clinical practice. International Journal of Obesity 29, 1153-67.

Government Office for Science. 2007: The foresight report tackling obesities: future choices. Retrieved 5 March 2009 from http://www.foresight.gov.uk/Obesity/20.pdf

Ham, C. 1990: Analysis of health policy - principles and practice. Scandinavian Journal of Social Medicine 46(suppl), 62-66.

House of Commons Health Committee. 2004: Obesity: third report of session 2003-2004. London: The Stationary Office.

Jain, A. 2005: Treating obesity in individuals and populations. The British Medical Journal 331, 1387-90.

Primary Health Care Research \& Development 2011; 12: 83-94 
Kopelman, P., Jebb, S.A. and Butland, B. 2007: Executive summary: Foresight 'Tackling Obesities: Future Choices' project. Obesity Reviews 8(Suppl 1), vi-ix.

London Health Observatory. 2008: Lifestyle \& behaviour obesity. Retrieved 25 July 2008 from http://www.lho.org.uk/ HIL/Lifestyle_And_Behaviour/Obesity.aspx

Miller, W.C., Koceja, D.M. and Hamilton, E.J. 1997: A metaanalysis of the past 25 years of weight loss research using diet, exercise or diet plus exercise intervention. International Journal of Obesity 21, 941-47.

Mulvihill, C. and Quigley, R. 2003: The management of obesity and overweight. An analysis of reviews of diet, physical activity and behavioural approaches. Evidence briefing, first edition. London: National Institute for Health and Clinical Excellence. Retrieved 15 July 2009 from http:// www.nice.org.uk/niceMedia/documents/obesity_evidence_ briefing.pdf

National Audit Office. 2001: Tackling obesity in England. London: The Stationery Office. Retrieved 25 July 2008 from http://www.nao.org.uk/publications/nao_reports/00-01/ 0001220.pdf.

National Health Services. 2008: Health Survey for England 2006 latest trends. From http://www.ic.nhs.uk/statistics-anddata-collections/healthand-lifestyles-related-surveys/healthsurvey-for-england/health-survey-for-england-2006-latest-trends

NICE. 2006: Obesity: guidance on the prevention, identification, assessment and management of overweight and obesity in adults and children. London: National Institute for Health and Clinical Excellence (updated 2006; cited 2009 27th July); National Institute for Health and Clinical Excellence clinical guideline 43. From http://www.nice.org.uk/ nicemedia/pdf/CG43NICEGuideline.pdf

Nield, L., Moore, H., Hooper, L., Cruickshank, K., Vyas, A. Whittaker, V. and CD NHS Hammersmith and Fulham. 2009: Dietetics. London, UK: National Health Service (updated 12 December 2009; cited 27 July 2009); From http://www.hfpct.nhs.uk/localservices/communityservices/ dietetics/index.asp

Norris, S.L., Zhang, X., Avenell, A., Gregg, E., Schmid, C.H., and Lau, J. 2005: Pharmacotherapy for weight loss in adults with type 2 diabetes mellitus. Cochrane Database of Systematic Reviews 2005, Issue 1. Art. No.: CD004096. DOI: 10.1002/14651858.CD004096.pub2. The Cochrane Library, 1-73. Retrieved 27 July 2009 from http://mrw.interscience. wiley.com/cochrane/clsysrev/articles/CD004096/frame.html

Orozco, L.J., Buchleitner, A.M., Gimenez-Perez, G., Roque i Figuls, M., Richter, B. and Mauricio, D. 2009: Exercise or exercise and diet for preventing type 2 diabetes mellitus. In Cochrane Database of Systematic Reviews 2008, Issue 3.
Art. No.: CD003054. DOI: 10.1002/14651858.CD003054.pub3: The Cochrane Library, 1-81. Retrieved 27 July 2009 from http://mrw.interscience.wiley.com/cochrane/clsysrev/articles/ CD003054/frame.html

Padwal, R., Li, S.K. and Lau, D.C.W. 2003: Long-term pharmacotherapy for overweightand obesity: a systematic review and meta-analysis of randomized controlled trials. International Journal of Obesity and Related Metabolic Disorders 27, 1437-46.

Picot, J., Jones, J., Colquitt, J.L., Godspodarevskaya, E., Loveman, E. and Baxter, L. 2009: The clinical effectiveness and cost-effectiveness of bariatric (weight loss) surgery for obesity: a systematic review and economic evaluation. Health Technology Assessment 13, 1-230.

Pryke, R. and Docherty, A. 2008: Obesity in primary care: evidence for advising weight constancy rather than weight loss in unsuccessful dieters. British Journal of General Practice 58, 112-17 (116).

Reedy, S. 2009: An evidence-based review of obesity and bariatric surgery. The Journal for Nurse Practitioners 5, 22-29.

Rodriguez-Garcia, R. 2000: Health policy in a nutshell. Washington, DC: The George Washington University Center for Global Health.

Salem, L., Jensen, C.C. and Flum, D.R. 2005: Are bariatric surgical outcomes worth their cost? A systematic review. Journal of the American College of Surgeons 200, 270-78.

Shaw, K.A., Gennat, H.C., O'Rourke, P. and Del Mar, C. 2006: Exercise for overweight and obesity. In Cochrane Database of Systematic Reviews 2006, Issue 4. Art. No.: CD003817. DOI: 10.1002/14651858.CD003817.pub3: The Cochrane Library, 1-33. Retrieved 27 July 2009 from http://mrw.interscience.wiley.com/cochrane/clsysrev/articles/ CD003817/frame.html

Summerbell, C.D., Waters, E., Edmunds, L., Kelly, S.A.M., Brown, T. and Campbell, K.J. 2005: Interventions for preventing obesity in children. In Cochrane Database of Systematic Reviews 2005, Issue 3. Art. No.: CD001871. DOI: 10.1002/14651858.CD001871.pub2: The Cochrane Library, 1-107. Retrieved 25 February 2010 from http://mrw. interscience.wiley.com/cochrane/clsysrev/articles/CD003817/ pdf_fs.html

World Health Organization. 1999: Health 21: the health for all policy framework for the WHO European region. In European Health for All Series; no. 6. Denmark: World Health Organization Regional Office for Europe Copenhagen.

World Health Organization. 2006: Obesity and overweight. Retrieved 16 June 2009 from http://www.who.int/ mediacentre/factsheets/fs311/en/index.html 